\title{
THE CLOSURE OF TYPES OF CONNECTED SETS
}

\author{
PAUL M. SWINGLE
}

All known examples in the plane of widely connected sets, finitelycontaining connected sets, and biconnected sets without dispersion point are such that their closures are indecomposable continua. Here we propose to give examples of these types of connected sets whose closures are domains. ${ }^{1}$ The imbedding space is the euclidean plane and, wherever needed, the hypothesis of the continuum and Zermelo's axiom are assumed.

THEOREM 1. In any connected domain, $D$, in the plane there exists a subset $T$ such that both $T$ and $D-T$ are indecomposable connexes and $T, D-T$, and $D$ all have the same closure.

Proof. To prove this we used the familiar "boring" process with modifications-by means of which $\mathrm{Wada}^{2}$ showed the existence of an indecomposable continuum in the plane-by omitting the interior of a "canal," $T^{\prime}$, where the width of $T^{\prime}$ is diminished a countable number of times, with limit zero, and so "cut" from $D$ that every point of $\bar{D}-T$ is a limit point of $T$. It is seen that any point of $D$ is contained in the interior of a circle, which itself is contained in $D$, for otherwise this point is a boundary point of $D$. Thus $D$ is covered by a set $(R)$ of circular regions. Since $D$ is separable, ${ }^{3}$ let $N=p_{1}$ $+p_{2}+\cdots$ be a set of points of $D$ such that $\bar{N}=\bar{D}$.

Since $D$ is connected, there exists a simple chain, ${ }^{4} C_{1}^{\prime}$, of the regions

\footnotetext{
Presented to the Society, April 28, 1950; received by the editors February 25, 1950.

1 These examples were obtained in considering the unsolved problem: If $C$ is an indecomposable connexe, does there exist a point $p$ of $\bar{C}$ such that $C+p$ is a decomposable connexe? See my paper, Indecomposable connexes, Bull. Amer. Math. Soc. vol. 47 (1941) p. 799. Hereafter this paper will be referred to as "Ind. Con."

Given a connected set $C$ : then $C$ is widely connected if $C$ and every connected subset of $C$ have the same closure; $C$ is an indecomposable connexe if for every two connected subsets $H$ and $K$, where $C=H+K$, either $C$ and $H$ or $C$ and $K$ have the same closure; $C$ is biconnected if $C$ does not contain two mutually exclusive nondegenerate connected subsets; $C$ is a finitely-containing connected set if, for every positive integer $n, C$ contains $n$ mutually exclusive nondegenerate connected subsets, but $C$ does not contain infinitely many such subsets; the connected subset $K$ is a proper connexe subclosure if $K$ and $C$ do not have the same closure.

2 See K. Yoneyama, The theory of continuous sets of points, Tôhoku. Math. J. vol. 12 (1917) p. 60.

${ }^{3}$ G. T. Whyburn, Analytic topology, Amer. Math. Soc. Colloquium Publications, vol. 28, 1942, Theorem 3.7, p. 4 .

4 R. L. Moore, Foundations of point set theory, Amer. Math. Soc. Colloquium Publications, vol. 13, 1932, Theorem 77, p. 56.
} 
of $(R)$ joining $p_{1}$ and $p_{2}$. Let $p_{i}=q_{i}(i=1,2)$, and let the point set contained in the regions of $C_{1}^{\prime}$ be $C_{1}$. Let $q_{3}$ be the point of lowest subscript in $N$ not contained in $\bar{C}_{1}$. Let $R_{1}$ be a region of $(R)$ which is such that $C_{1}^{\prime}+R_{1}$ is a simple chain from $q_{1}$ to a point $q_{2}^{\prime}$ of $D$ not contained in $C_{1}$. Let $r_{1}$ be the radius of the smallest circle which is the boundary of a region of $C_{1}^{\prime}+R_{1}$, and let $(R)_{1}$ be the set of regions of $(R)$, contained in $D-\bar{C}_{1}$, whose boundaries are circles with radius less than or equal to $r_{1} / 2$. Then there exists a simple chain of regions of $(R)_{1}, C_{2}^{\prime \prime}$ say, joining $q_{2}^{\prime}$ and $q_{3}$ and there exists a simple chain, $C_{2}^{\prime}$ of $C_{1}^{\prime}+R_{1}+C_{2}^{\prime \prime}$, which joins $q_{1}$ and $q_{3}$ and contains $q_{2}$ in one of its regions.

Thus, continuuing in this manner, it is seen that there exist simple chains $C_{1}^{\prime}, C_{2}^{\prime}, C_{3}^{\prime}, \cdots$, where $C_{j}$ is the point set contained in the regions of $C_{j}^{\prime}(j=1,2, \cdots), C_{j}^{\prime}$ joins $q_{1}$ and $q_{j}$ where $q_{j}$ is the point of $N$ of lowest subscript not contained in $\bar{C}_{j-1}$. Also $q_{1}+q_{2}+\cdots+q_{j}$ is contained in $C_{j}$. Let $r_{j}$ be the radius of the smallest circle bounding a region of $C_{j}^{\prime}$. It is seen that $r_{1}, r_{2}, \cdots$ may be taken with zero as sequential limit. Let $T_{1}=C_{1}+C_{2}+\cdots$. Thus $T_{1}$ is a connected domain and it is seen without difficulty, by familiar methods, that $\bar{D}-T_{1}=I_{1}$ is an indecomposable continuum.

Since the proof above is valid for any connected domain, it follows that there exist connected domains $T_{1}, T_{2}, \cdots$, where $T_{j+1}$ is contained in $T_{j}, \bar{D}-T_{j}$ is an indecomposable continuum, and $T_{j}$ consists of the interiors of a countable number of circles of class $(C)_{j}$ say. Let $r_{j}^{\prime}$ be the radius of the largest circle of $(C)_{j}$. It is evident that $r_{1}^{\prime}, r_{2}^{\prime}, \cdots$ can be taken converging to zero. Let $T=T_{1} \cdot T_{2} \cdot T_{3} \cdots \cdots$ It is seen easily that $T$ and $D-T$ are both connected ${ }^{5}$ and, by the same methods used to show that Wada's continuum is indecomposable, it is seen that $T$ and $D-T$ are both indecomposable connexes. 6

Theorem 2. In any connected domain, $D$, in the plane there exists a widely connected set, $W$, such that $\bar{W}=\bar{D}$.

Proof. From the proof of Theorem 1 we have the set of inde-

5 R. L. Moore, loc. cit., Theorem 41, p. 27. See also B. Knaster and C. Kuratowski, Sur les ensembles connexes, Fund. Math. II, p. 253.

- The more difficult case is for $D-T$. Under the supposition that $D-T=H+K$, $H, K$ connexes, $\bar{H} \neq(D-T)^{-} \neq \bar{K}$, there exist regions $R_{h}$ and $R_{k}$, where $R_{h} \cdot K=0$, $R_{k} \cdot H=0, h \in H$, and $k \in K$. For infinitely many $j$ 's there exists a simple chain, $K_{i}$, of a finite number of the regions making up $T_{i}$, where $K_{j}$ makes a "loop" from $R_{h}$, say, enclosing points of $R_{k}$. These simple chain "loops," $K_{i}$, can be taken so that the common part of the $\bar{K}_{j}$ 's is a subcontinuum of $T$, which separates the set $R_{k} \cdot(D-T)$, giving a contradiction to the supposition $D-T$ is decomposable. Use may be made, loc. cit., of Moore's Theorem 42, p. 28 or Theorem 80, p. 58. $(D-T)^{-}$denotes the closure of $(D-T)$. 
composable continua $I_{1}, I_{2}, \cdots$, where $I_{j}=\bar{D}-T_{j}, I_{j} \subset I_{j+1}, T_{j}$ is dense in $I_{k}, k<j$, and $T$ is dense in $I_{j}(j=1,2, \cdots)$. Let $C_{1}, C_{2}, \cdots$ be a set of concentric circles about a point of $T$ so taken that $C_{j}$ separates $I_{j}$. Thus each $C_{j}$ contains points of uncountably many composants ${ }^{7}$ of $I_{j+1}$. By the axiom of choice we shall choose the points of $W$ so that no point of $C_{j} \cdot W$ is contained in $I_{j}$. The class $(K)$ of separating continua of $\bar{D}$ is known to be of the power, $c$, of the continuum. Each element $K$ of $(K)$ separates all except a finite number of the indecomposable continua $I_{j}$, if $K$ is in $T_{o}, K$ does not separate $I_{1}$, $I_{2}, \cdots, I_{o-1}$. Thus for all except a finite number of the $I_{k}$ 's, $K \cdot I_{k}$ contain points of an uncountable number of composants of $I_{k}$.

Let $(H)_{j}$ be the set of composants of $I_{j}$, except for the composants which contain points of the circular boundaries of a $T_{i}(i=1,2, \cdots)$. Let $(H)_{j}^{\prime}$ and $(H)_{j}^{\prime \prime}$ be a division of $(H)_{j}$ into two mutually exclusive subclasses each of power $c$, since any set of power $c$ can be divided in this manner.

Let $(C)_{j}$ be the subclass of elements of $(K)$, each of which is a subset of $C_{j}+T$. The class $(C)_{j}$ can be put into a 1-1 correspondence, $t_{j}$, with a subclass of $(H)_{j+1}^{\prime}$. Let $(p)_{j}$ be a class of points, one each chosen from each $C^{\prime} \cdot H_{j+1}^{\prime}$, where $C^{\prime}$ and $H_{j+1}^{\prime}$ are the two elements which correspond under $t_{j}$, it having been noted above that $C^{\prime} \cdot H_{j+1}^{\prime}$ is nonvacuous.

Let $(K)^{\prime}$ be the subclass of $(K)$, each element of which does not contain a point of a $(p)_{j}$. Then $(K)^{\prime}$ can be put into a 1-1 correspondence, $t_{j}$, with a subclass of $(H)_{j}^{\prime \prime}$. Let $(q)$ be the class of points, $q$, one each being chosen for each $K^{\prime}$ of $(K)^{\prime}$ from a $K^{\prime} \cdot H_{i}^{\prime \prime}$, where $K^{\prime}$ and $H_{i}^{\prime \prime}$ are the elements corresponding under a $t_{j}$ and $K^{\prime} \cdot H_{i}^{\prime \prime} \neq 0$.

Let $W=(q)+(p)_{1}+(p)_{2}+\cdots$. Then $W$ is connected, since each separating continuum, an element of $(K)$, contains a point of $W$. Furthermore there does not exist an $I_{j}$ such that $W \cdot I_{j}$ contains a connected subset $W^{\prime}$ dense in $I_{j}$, for $C_{j}$ separates $I_{j}$ but $W^{\prime} \cdot C_{j}=0$. Also no composant of an $I_{j}$ contains a nondegenerate connected subset, since at most one point was chosen from any one composant of $(H)_{j}$.

Suppose now that $W$ contains a subconnexe $V$ such that $\bar{V} \neq \bar{W}$. Then there exists a region $R$ of $D$ such that $\bar{R} \cdot \bar{V}=0$. Since no $I_{j}$ contains $V$, it follows that $T$ must contain a limit point, $u$, of $V-$ since there must exist a compact "loop" of a $T_{j}$ "from and to" $R$ which contains $V$. Thus for all but a finite number of the $T_{j}$ 's there exists a subchain, $B_{j}$ say, of $T_{j}$ joining two mutually exclusive sub-

${ }^{7}$ R. L. Moore, loc. cit., Theorems 106, 110, pp. 75-77, and Z. Jamiszewski and C. Kuratowski, Sur les continus indecomposables, Fund. Math. I, pp. 219-220. Compact $I_{j}$ 's always can be used. 
continua of the boundary of $R$ and such that $B_{j}$ contains $u$ and has its boundary in $I_{j}+$ (boundary of $R$ ). It is to be noted that the part of the boundary of $B_{j}$ not in $\bar{R}$ is in a composant of $I_{j}$ which contains no point of $W$. Since $u$ is a limit point of points not contained in any one $I_{j}$, it follows that there exists a $B_{j}$, infinitely many in fact, whose boundary in $I_{j}$ together with part of the boundary in $R$ separates $V$. Therefore $V$ is not connected and so $W$ is widely connected and $\bar{W}=\bar{D}$.

TheOREM 3. Any connected domain, $D$, in the plane contains a widely connected subset, $B$, such that $B$ is a biconnected set without dispersion point and $\bar{B}$ contains a subdomain of $D$.

Proof. Since each point of $D$, as noted above, must be contained in the interior of a circle, let $A B C D$ be a square that lies interior to a circle contained in $D$. Let $P$ be a Cantor ternary set constructed upon $A B$ and through each point $p$ of $P$ pass a straight line interval $p q$ to a point $q$ of $C D$, parallel to $B C$. Let $H_{1}, H_{2}, \cdots$ be the set of rectangles having intervals $p q$ as sides and "omitted third intervals" on $A B$ as bases. In the proof of Theorem 1 take the canals $T_{1}, T_{2}, \ldots$ so as not to pass through $H_{1}, H_{2}, \cdots$-it is not difficult to see that this can be done by modifying slightly the proof of Theorem 1 . Thus the set $T$, of the proof of Theorem 1, is now contained in $D$ $-\left(H_{1}+H_{2}+\cdots\right)$ and $\bar{T}$ contains the set of perpendiculars in $A B C D$ constructed upon $P$. Thus we have exactly the case considered by E. W. Miller in the lemma of his paper, Concerning biconnected sets. ${ }^{8}$ Thus following Miller's proof and that of Theorem 2 above we can obtain in $D-\left(H_{1}+H_{2}+\cdots\right)-T$ a widely connected set, $B$, which is also a biconnected set without dispersion point, and such that $\bar{B}=\left(D-\left(H_{1}+H_{2}+\cdots\right)\right)-$. To prove that $B$ is widely connected we need to follow the proof of Theorem 2 above, but otherwise Miller's proof is followed.

Since Miller uses his nowhere dense perfect set $P$ in the proof of his Theorem 8 only to make sure he can choose his point $P_{\beta}$ of $B_{\beta}$, where $B_{\beta} \cdot \Delta=0$ (pp. 131-133), it is seen in that proof that this is not essential to his proof-he uses $P$ only because indecomposable continua of that type were known. Thus we can take a square $A B C D$ in $D$ and run our canals $T_{1}, T_{2}, \cdots$ through it, with sides parallel to $B C$, otherwise as in Theorem 1 above. Thus we obtain a $T$, of the

${ }^{8}$ Fund. Math. XXIX (1937) p. 127. Miller was interested in the problems whether a biconnected set without a dispersion point had to have a biconnected subset contained in an indecomposable continuum, which we here answer, and whether it had to be in a widely connected set, still an unsolved problem. See "Ind. Con.," Example A and footnote, p. 776. 
proof of Theorem 1, running through $A B C D$ parallel to $B C$, and such that $\bar{T}=\bar{D}$. Here $P=A B-(A B \cdot T)$, for the $P$ of Miller's proof. Thus proceeding exactly as above we obtain the proof of the following theorem.

TheOREM $3^{\prime}$. Any connected domain, $D$, in the plane contains a widely connected set, $B$, which is also a biconnected set without dispersion point, and such that $\bar{B}=\bar{D}$.

By seeing to it that the set $T$ of the proof of Theorem 1 above has in its limiting set the "triangle set" needed by Knaster and Kuratowski to show the existence of a biconnected set with dispersion point, ${ }^{9}$ we can see easily that $B$ can be constructed in Theorem $3^{\prime}$ such that there exists a point $p$, the dispersion point of the Knaster and Kuratowski biconnected set, where $B+p$ is the sum of two mutually exclusive biconnected sets, one with dispersion point and the other without. Thus this gives an example of a widely connected set, $B$, having the property that there exists a point $p$ of $\bar{B}$ such that $B+p$ is not widely connected. ${ }^{10}$

TheOREM 4. Any connected domain, $D$, in the plane contains a widely connected subset, $F$, which is also a finitely-containing connected set, and such that $\bar{F}=\bar{D}$.

Proof. We proceed exactly as in the proof in my paper, $A$ finitelycontaining connected set, ${ }^{11}$ except that we base the present proof upon the biconnected set of Theorem $3^{\prime}$ rather than upon Miller's set. Here the set, $T$, of the proof of Theorem 1 above, will run "parallel" to a side of the squares, $Q, Q_{1}, Q_{2}, \cdots$ which were needed in that proof, while in these squares.

Theorem 5. If $C$ is an indecomposable connexe, $K$ an indecomposable continuum, $\bar{C}=\bar{K}$, then there does not exist a point $p$ such that $C+p$ is a decomposable connexe.

Proof. Suppose that there exists $p$ such that $C+p$ is a decomposable connexe, that is, there exist proper connexe subclosures $U$ and

- Loc. cit., Fund. Math. II, Example $\delta$, p. 250.

10 Unsolved problems on widely connected sets are: If $W$ is widely connected, but there exists a point $p$ such that $W+p$ is not widely connected, is $W+p$ always an indecomposable connexe; If $W$ is widely connected does there always exist a point $q$ such that $W+q$ is widely connected. For all known examples of widely connected sets the answer to both is in the affirmative. A similar answer is true for the question: If $I$ is an indecomposable connexe, does there always exist a point $p$ such that $I+p$ is an indecomposable connexe, $I$ not closed.

${ }^{11}$ Bull. Amer. Math. Soc. vol. 46 (1940) pp. 178-181. 
$V$ of $C+p$, such that $C+p=U+V$. Since $(C+p)^{-}=K$ and $\bar{U} \neq K$ $\neq \bar{V}$, but $K=\bar{U}+\bar{V}$, it follows that $K$ is decomposable. Thus the theorem is true.

The proof of the following theorem follows at once from the definitions involved.

THEOREM 6. A necessary and sufficient condition that a closed set be an indecomposable continuum is that it be an indecomposable connexe.

Definition 1 . The connexe $M$ is indecompoundable if and only if for every two mutually exclusive subconnexes $H$ and $K$, where $M$ $=H+K$, either $H$ and $M$ or $K$ and $M$ have the same closure.

Obviously an indecomposable connexe is indecompoundable. However a biconnected set is indecompoundable but need not be indecomposable. An example is not known of an indecompoundable connexe which is not biconnected.

Theorem 7. Let $C$ be an indecomposable connexe and $C+p$ be a decomposable connexe, $p$ a point. Let $U$ and $V$ be proper connexe subclosures such that $C+p=U+V$. Then $p \in U \cdot V, U-p$ and $V-p$ are each disconnected, and $U$ and $V$ are decomposable.

Proof. By Lemma $A^{\prime}$ of "Ind. Con.," ${ }^{12}$ if we assume $p$ is not an element of $U, C-U$ is connected. But then $C$ is the sum of the two proper connexe subclosures, $U$ and $C-U$, and so $C$ is decomposable. Thus $p \in U \cdot V$.

If $U-p$ is connected, also by Lemma $A^{\prime}$ of "Ind. Con," we see that $C-(U-p)$ is connected and so $C$ is the sum of two proper connexe subclosures, $U-p$ and $C-(U-p)$, contradicting the hypothesis that $C$ is indecomposable.

Hence let $U-p=U_{1}+U_{2}$ mutually separate. Then $U$ is the sum of the two proper connexe subclosures $U_{1}+p$ and $U_{2}+p$ and so $U$ is decomposable as is also $V$.

CoRollary 1. Under the hypothesis of Theorem 7, if $K$ is a subconnexe of $U-p$ or of $V-p$, then $K$ is a connexe of condensation of $C$ and $\bar{K}$ is a continuum of condensation of $\bar{C}$.

Proof. This follows by Theorems $108^{\prime}$ and $108^{\prime \prime}$ of "Ind. Con."

Corollary 2. If $C$ is an indecomposable connexe and $C+p$ is a decomposable connexe, $p$ a point, then $C+p$ is an indecompoundable connexe.

\footnotetext{
12 See loc. cit., p. 799. Lemma $\mathrm{A}^{\prime}$ states: If $M$ is an indecomposable connexe and $N$ is a proper connexe subclosure of $M$, then $M-N$ is connected.
} 
Proof. By Theorem $7, C+p$ is not the sum of two mutually exclusive proper connexe subclosures $U$ and $V$, for $p \in U \cdot V$. Thus the corollary follows by Definition 1.

CoROLlary 3. Under the hypothesis of Theorem 7,C+p is the composant of ${ }^{13} C+$ which contains $p$.

Proof. Since $C+p$ is decomposable, there exist proper connexe subclosures $U$ and $V$, where $C+p=U+V$. By Theorem $7, p \in U \cdot V$ and so every point of $U$, and every point of $V$, is contained in a proper connexe subclosure of $C+p$ containing $p$. Hence $C+p$ is contained in the composant of $C+$ which contains $p$.

Theorem 8. If the connexe $M$ is indecompoundable and decomposable, $M$ being the sum of two proper connexe subclosures $A$ and $B$, then $M-A$ is disconnected.

Proof. This follows at once from the definitions involved.

Theorem 9. If $C$ is an indecomposable connexe, $N$ is a nonvacuous subset of $\bar{C}$ such that, for each point $p$ of $N, C+p$ is decomposable, then $C+N$ is decomposable.

Proof. Since for $p$ of $N, C+p$ is decomposable, there exist proper connexe subclosures $U$ and $V$, whose sum is $C+p$. But $(C+p)^{-}=\bar{C}$ and, for each point $q$ of $N-p, q \subset \bar{C}$. Hence $q \subset \bar{U}$ or $q \subset \bar{V}$. Say $N_{u}$ of $N$ is contained in $\bar{U}$ and $N_{v}$ of $N$ is contained in $\bar{V}$. Then $C+N$ is the sum of the two proper connexe subclosures $U+N_{u}$ and $V+N_{v}$ and so $C+N$ is decomposable.

It is not known whether the set $C+p+q$, in Theorem 10 below, exists.

Theorem 10. Let $C, C+p, C+q$ be indecomposable connexes, $p, q$ points, and suppose $C+p+q$ is a decomposable connexe which is the sum of the two proper connexe subclosures $X$ and $Y$. Then $p+q \subset X \cdot Y$ and $X-p, X-q, Y-p, Y-q$ are all disconnected.

Proof. It follows from Theorem 7 that $p+q \subset X \cdot Y$. Suppose $X-p$ is connected. Then, by Lemma $\mathrm{A}^{\prime}$ of "Ind. Con," $C+q$ is decomposable since it is the sum of the two proper connexe subclosures $X-p$ and $(C+q)-(X-p)$.

Of interest in connection with the example following Theorem $3^{\prime}$ is the following theorem.

TheOREM 11. Let $W$ be widely connected, $W+p$ be connected but not

\footnotetext{
${ }^{18}$ See Definition in "Ind. Con.," loc. cit., p. 797.
} 
widely connected, $p$ a point. Then there exists a connected subset $B$ of $W+p$, where $\bar{B} \neq \bar{W}, p \in B, B-p$ is disconnected and $B$ is biconnected.

Proof. Since $W+p$ is not widely connected, by definition, there exists a proper connexe subclosure $B$ of $W+p$. If $B \subset W, \bar{B}=\bar{W}$. Hence $p \in B$. If $B-p$ contains a subconnexe $B^{\prime}$, then $\bar{B}^{\prime}=\bar{W}$. Therefore $B-p$ is totally disconnected and so $B$ is biconnected, having dispersion point $p$.

TheOREM 12. Let $C$ be an indecomposable connexe, $p \in C, R$ a region. If $p \in R$, then $C \cdot R$ is disconnected and, if $p \in C-C \cdot R$, then $C-C \cdot R$ is disconnected.

Proof. This follows from Lemma A' of "Ind. Con."

Theorem 13. If $C$ is an indecomposable connexe, then $C$ is not the sum of a finite number of proper connexe subclosures.

Proof. Suppose $C$ is the sum of the proper connexe subclosures $C_{1}, C_{2}, \cdots, C_{n}$. Since $C$ is connected one of the sets $C_{1}$ and $\left(C_{2}+C_{3}+\cdots+C_{n}\right)$ contains a limit point of the other and it follows that either $C_{1}$ contains a limit point of a $C_{j}(j=2,3, \cdots, n)$ or a $C_{j}$ contains a limit point of $C_{1}$. Therefore $C_{1}+C_{j}$ is connected. Repeating this process, with $C_{1}+C_{j}$ in place of $C_{1}$, one sees that we may take our subscripts, to conserve notation, such that, for each $t<n, C_{1}+C_{2}+\cdots+C_{t}$ is connected.

Since $\bar{C}_{n} \neq \bar{C}$, it follows that $C_{1}+\cdots+C_{n-1}$ and $C$ have the same closure. Hence, by Theorem A of "Ind. Con.," $C_{1}+\cdots+C_{n-1}$ is an indecomposable connexe. One can repeat this argument, obtaining finally that $C_{1}+C_{2}$ is an indecomposable connexe with the same closure as $C$. Since this gives a contradiction, the theorem is true.

COROLLARY 4. Under the hypothesis of Theorem 7 , for every $n$, either $U-p$ or $V-p$ is the sum of $n$ mutually separate subsets.

Proof. This follows from Theorems 7 and 13.

TheOREM 14. Let $C$ be a decomposable connexe and such that, for every three subconnexes $U, V, W$ where $C=U+V+W$, one of these has the same closure as $C$. Then $C$ is the sum of two indecomposable connexes. If $C$ is a decompoundable connexe and $U, V, W$ are also mutually exclusive, then $C$ is the sum of two mutually exclusive indecompoundable subconnexes.

Proof. This follows easily from the definitions involved.

The University of Miami 